\title{
Lebensqualitätsmessung mit „Selbsteinschätzung der Lebensqualität (SAQOL)“ bei Patienten mit Bronchialkarzinom und COPD: Lebensqualität hat Einfluss auf Überlebenszeit
}

\author{
P. R. Grahmann ${ }^{1,2}$ \\ A. J. Crockett ${ }^{3}$
}

\author{
Self Assessment Quality of Life and Lung Diseases (SAQOL in Patients \\ with Pulmonary Carcinoma: Influence to Survival and Impact of Chronic \\ Obstructive Pulmonary Disease
}

\section{Zusammenfassung}

Einleitung: Bronchialkarzinom (BC) und chronisch obstruktive Lungenerkrankung treten oft gemeinsam auf. Es gibt bereits verschiedene Fragebogen für COPD-Patienten, die für den deutschen Gebrauch übersetzt wurden, es besteht jedoch Bedarf nach einem kurzen Instrument, mit dem Auswirkungen begleitender Erkrankungen oder Interventionen auf die Lebensqualität erfasst werden können, besonders in deutscher Sprache. Da es bis heute keinen deutschen Fragebogen für die Lebensqualität von Patienten mit Lungenerkrankungen gibt, besonders für die beiden häufigsten Erkrankungen, BC und die oft begleitende COPD, wurde ein entsprechendes Instrument generiert und eingesetzt. Methoden: Die Machbarkeit und Validität eines kurzen, durch Patienten selbst auszufüllenden Lebensqualitätsfragebogen für COPD und pulmonale Neoplasien wurde evaluiert. Die 27 Fragen zur Messung der Lebensqualität des „Selbsteinschätzung der Lebensqualität (SAQOL)“, zusammengefasst in 5 Domänen, wurden an ambulante Patienten mit Lungenkrebs ausgegeben (18 Frauen, 75 Männer, Alter 18 bis 81, im Mittel 62,6 Jahre), davon litten 54 (58,1\%) auch unter COPD. Die Patienten füllten auch einen etablierten Tumorfragebogen der „European Organization for Research and Treatment of Cancer“ (EORTC: QLQ-C30) aus. In einer weiteren Gruppe von Patienten $(n=20)$ ohne Malignom mit COPD und Sauerstofflangzeittherapie wurde mit australischen Patienten die englische Version von SAQOL gegen das Nottingham Health Profile (NHP) verglichen. Ergebnisse: Die Korrelationen von SAQOL zu den vergleichbaren Domänen des tumorbezo-

\section{Abstract}

Background: Lung cancer and chronic obstructive pulmonary disease (COPD) often occur concomitantly. Whilst there are several questionnaires relating to COPD that have been translated into German (CCOPDQ, CRQ, SGRQ) there is a need for a short instrument measuring the impact of the concomitant diseases or intervention on a patient's Quality of Life specifically in German language. As there is to date no genuine German questionnaire neither in general, nor in detail for both, lung cancer and the often concomitant COPD, we created and tested an appropriate single instrument. Methods: The feasibility and validity of a short, self-administered quality of life questionnaire for use with patients diagnosed with COPD and/or pulmonary malignancies was evaluated. The 27 questions of Self Assessment Quality of Life in Lung Diseases (SAQOL) questionnaire were issued to out-patients with lung cancer (18 females, 75 males, mean 62.6 years, 18 to 81), 54 of with concomitant COPD. Results: Correlations to comparable domains of the tumour-related QLQ-C30 of EORTC were good in cancer patients. Quality of life impact scores in the shorter and organ specific SAQOL domains showed even higher values than the QLQ C-30. A higher quote of Quality of life impaction was related to a poorer prognosis despite comparable tumour stages. There was a relevant impact related to the presence of COPD in addition to lung cancer. To investigate the validity in severe COPD an English version of the questionnaire was applied in the Respiratory Unit, Adelaide Australia, together with the Nottingham Health Profile in patients with long-term

${ }^{1}$ Pneumologie und Allergologie/Immunologie, Friedrich-Schiller-Universität, Jena

${ }^{2}$ Bereich Pneumologie der Abteilung Thoraxchirurgie, Albert-Ludwigs-Universität, Freiburg

${ }^{3}$ Primary Care Respiratory Unit, Department of General Practice, University of Adelaide, Adelaide, South Australia 
genen QLQ-C30 der EORTC waren bei den Tumorpatienten gut. Das Ausmaß der Beeinträchtigung wurde in dem kürzeren und organspezifischen SAQOL deutlicher abgebildet als in dem QLQ-C30. Zudem war eine schlechtere Lebensqualität mit einer kürzeren Überlebenszeit verbunden, trotz vergleichbarer (inoperabler, inkurabler) Tumorstadien. Weiterhin waren Vorhandensein und Ausprägung einer COPD ein wichtiger Faktor der Beeinträchtigung der Lebensqualität. Dieser Faktor wurde besser mit dem SAQOL abgebildet. Um hier die Validität gegen einen genuin für COPD evaluierten Fragebogen zu messen, wurde eine englische Version des SAQOL (neben anderen Fragebogen), ausgefüllt von australischen Patienten, zusammen mit dem Nottingham Health Profile verglichen. Bei den 20 Patienten mit schwerer COPD und Sauerstofflangzeittherapie ohne Neoplasie ließ sich eine sehr gute Korrelationen in den COPD-relevanten Domänen zeigen. Schlussfolgerung: Wir können feststellen, dass SAQOL ein einfach einzusetzender Lebensqualitätsfragebogen ist, der zum einen gute Korrelationen zu einem etablierten und verlässlichen Tumorfragebogen aufweist, der aber auch zum anderen sich sehr gut mit einem validen lungenspezifischen COPD Fragebogen vergleichen lässt. Zudem konnte eine Assoziation schlechter eingestufter Lebensqualität mit einer kürzeren Überlebenszeit belegt werden, bei sonst gleichen Ausgangsbedingungen eines weit fortgeschrittenen Tumorstadiums. oxygen therapy ( $\mathrm{n}=20$ ), which correlated well with the lung specific domains of SAQOL. Conclusion: We conclude that SAQOL is an easy to handle questionnaire, which shows on the one hand good correlation to the tumour-related domains of the broadly investigated and reliable QLQ C30, and on the other hand to the long-term used and valid lung specific generic Nottingham Health Profile in COPD. The important influence COPD in quality of life impact highlights the need for an organ specific questionnaire for both, lung cancer and obstructive airway disease. This important factor for quality of life alteration seems to be underestimated in other generic questionnaires or it needs the issue of at least two different and more time consuming instruments for adequate assessment. Additionally a high degree of quality of life impaction was linked to a short survival in our study patients with advanced lung cancer.

\section{Hintergrund}

Die Verbindung von Zigarettenrauchen zur Ätiologie von sowohl Lungenkrebs als auch chronisch obstruktiver Lungenerkrankung (COPD) ist gut belegt [1,2]. Auch das Merkmal „Atemwegsobstruktion" ist gegenüber der Normalbevölkerung verknüpft mit einem vier- bis sechsfach erhöhten Risiko Lungenkrebs zu entwickeln, unabhängig von einer Raucheranamnese [3,4]. Es besteht die Notwendigkeit eines einfach zu handhabenden Fragebogens, der es im täglichen Umgang mit dem Patienten ermöglicht, objektiv die ärztlichen und medikamentösen Maßnahmen und ihre Auswirkungen auf die Lebensqualität abzubilden [5,6]. Solch ein Instrument sollte eine valide Erhebung der Selbsteinschätzung von Wohlgefühl und Stimmung ermöglichen. Bekannt ist eine Differenz zwischen der Fremdeinschätzung der Lebensqualität des Patienten durch medizinisch Tätige und demgegenüber der Selbsteinschätzung eines Patienten. In der Fremdeinschätzung werden regelhaft Symptome und objektive Befunde als Anhalt herangezogen. Dagegen ist die eigene Bewertung der Lebensqualitäteinschränkung durch den Patienten häufig ein besserer Maßstab für die tatsächlichen Auswirkungen auch schwerer Symptome auf den Altag [7].

Zusätzlich zu der klinischen Konstellation mit Symptomen und messbaren Parametern, bedarf es im Rahmen der Behandlung und Pflege einer chronischen Erkrankung eines Vehikels zur Abbildung der Stimmung und Konstitution. Diese Einschätzung sollte über ein Instrument standardisiert sein und quantifizierbar den Grad emotionaler, psychosozialer und physischer Lebensqualität ermöglichen. Es gab bereits viele Versuche, hierfür Fragebogen zu etablieren. Leider haben solche Instrumente häufig den Nachteil eines recht umfangreichen Fragekatalogs und sind zudem meist auf den Einsatz im Rahmen von Studien begrenzt, zudem benötigen sie nicht selten die Kompetenz eines professionellen Sozialwissenschaftlers für Anwendung und Auswertung. Ein weltweit häufig eingesetzter Fragebogen zur Ermittlung der gesundheitsbezogenen Lebensqualität wurde zunächst lediglich zur Risikostratifizierung von Versicherungsunternehmen generiert und dann posthoc nach millionenfachem Einsatz auf die betreffenden Organsysteme und Diagnosen zurückgeschlossen. Hier muss besonders der SF-36 genannt werden, der wesentliche psychosoziale Lebensqualitätseinschränkungen nicht erfassen kann und auch in der komprimierten Fassung des SF-12 nicht verlässlicher erscheint. Letztlich müssen SF-36 und SF-12 trotz ihres weitverbreiteten Einsatzes überwiegend in Multizenterstudien gerade bei pneumologischen Erkrankungen als unspezifische, faktor-extrahierte, posthoc evaluierte Instrumente für eine generelle gesundheitsbezogene Lebensqualitätsmessung gewertet werden, nicht dagegen als spezifische Instrumente. Zumeist bedarf es daher des zusätzlichen Einsatzes eines organspezifischen Fragebogens.

Ein geeigneter kurzer Fragebogen sollte durch den Patienten akzeptiert sein und durch den Kliniker in der täglichen Routine eingesetzt werden können. Solch ein Fragebogen sollte darüber hinaus von Patienten mit respiratorischen Erkrankungen generell, mit oder ohne Malignom verwendet werden können und dabei verlässliche Ergebnisse liefern. Mit diesen Vorgaben wurde ein Fragebogen generiert, der in seiner letzten evaluierten Fassung 27 Fragen in 5 Domänen vereinigte. Dieser Fragebogen entstand aus einer umfangreicheren Liste von Fragen, die ambulanten Patienten mit der Bitte um Bewertung und Gewichtung vorgelegt und durch Rückmeldung ergänzt wurde. 
Ein spezifischer Fragebogen wurde benötigt, da es keinen verfügbaren kurzen Lebensqualitätsfragebogen für deutschsprachige Patienten gibt, der den eingangs genannten Anforderungen genügt. Ins Deutsche direktübersetzte Fragebogen konnten die physische Belastung der Lebensqualität durch Malignome nicht adäquat wiedergeben $[7,8]$ oder waren nicht spezifisch genug für die Quantifizierung organinduzierter psychischer Alteration. [9-11]. Zudem bestehen landes- und kulturtypische Unterschiede in der subjektiven Einschätzung der Lebensqualität. Grundsätzliche Unterschiede können zum Beispiel in der besonderen Bedeutung als funktionierender Bestandteil in der Gesellschaft Nordamerikas gesehen werden und einer deutlich geringeren Gewichtung einer persönlichen Beeinträchtigung durch Emotion und Psyche [9]. Kürzlich in das Deutsche angepasste Fragebogen zielen zudem isoliert auf Atemwegserkrankungen und sparen pulmonale Malignome aus [12]. Dies führte zur Entwicklung eines spezifisch strukturierten, vom Patienten selbst auszufüllenden Fragebogen für Patienten mit pulmonalen Tumoren und Patienten mit COPD, was häufig miteinander kombiniert ist.

Als wir den Fragebogen einsetzten, wollten wir wissen, ob er einfach zu handhaben ist (in kurzer Zeit auszufüllen, akzeptabel für Patient und Arzt), valide für Tumorpatienten (mit einer ausreichend guten Korrelation zu einem etablierten tumorbezogenen Fragebogen) und verlässlich bei COPD Patienten (korreliert zur Lungenfunktion und einem spezifischen Fragebogen für chronische Atemwegserkrankungen). Zudem wurde der Fragebogen genutzt, um den Einfluss der Lebensqualität auf das Überleben in fortgeschrittenen Tumorstadien zu ermitteln. Eine Teil-Gruppe der Patienten $(n=54)$ mit fortgeschrittenem Bronchialkarzinom nahm an einer Multizenterstudie der EORTC teil [13]. Der andere Fokus lag auf der Auswirkung der COPD, auf die Lebensqualität von Patienten mit Lungentumor.

\section{Methoden}

\section{SAQOL-Fragebogen, Konstruktion und Domänen Fragepool und Endfassung des Fragebogens}

Um die verschiedenen Interessensbereiche des zukünftigen Fragebogens festzustellen, wurde ambulanten Patienten der Abteilungen Pneumologie und Lungenchirurgie der Albert-Ludwigs Universität, Freiburg ein offener Fragebogen ausgehändigt, mit zunächst nur der einzigen Frage nach für die Patienten wichtige Faktoren der Lebensqualität. Die in einer mehrwöchigen Periode gesammelten Faktoren wurden durch Patienten im nächsten Durchgang gewichtet. Den durch Häufigkeit der Nennung gelisteten Faktoren wurden Kategorien zugeordnet. Im nächsten Schritt wurden die formulierten Fragen vorgelegt und um Beantwortung und erneute Gewichtung gebeten. Es sollten Faktoren identifiziert werden, die für die COPD und für das Management des Lungenkarzinoms wesentlich sind. Diesem Rohfragebogen wurden die innerhalb der $90 \%$ Perzentile am häufigsten genannten Fragen entnommen. Der Fragebogen der Europäischen Arbeitsgemeinschaft zur Erforschung und Behandlung des Krebses (QLQ-C 30 der EORTC) $[13,14]$ wurde ursprünglich für den Fragebogenaufbau als Vorlage gedacht, erwies sich aber im Weiteren unter den genannten Zielen als nicht ausreichend. So fehlen dort zum Beispiel die von den Patienten als wichtig eingestuften Fragen nach Alteration der Sexualität und Spiritualität. Von ei- nem umfänglicheren Set wurde durch Patientenvoten letztlich die extrahierte letzte Version generiert, von der aus eine weitere Verkürzung zu Informationsverlust geführt hätte. Der hier vorgestellte Fragebogen zur Selbstmessung der Lebensqualität (SAQOL, siehe Anhang) folgt einem multidimensionalen Konzept. Lebensqualität wurde spezifiziert als solche Domänen, die durch Patienten im Rahmen der Behandlung und Versorgung von Bronchialkarzinom (BC) oder/und COPD identifiziert oder vorgeschlagen wurden. Der Fragebogen konzentriert sich somit auf Aspekte der gesundheitsbezogenen Lebensqualität und umfasst zudem die Frage nach der generellen Lebensqualität. Der Fragebogen umfasst folgende Domänen: Physische Aspekte, wie Schmerz und weitere Symptome; funktionelle Möglichkeiten; familiäres und emotionales Wohlgefühl, Spiritualität; Zufriedenheit mit der Behandlung, finanzielle Auswirkungen der Erkrankung; Zukunftsorientierung; Sexualität, soziales und berufliches Funktionieren.

Die Fragen wurden auf den Zeitraum der letzten vier Wochen bezogen. Die Symptome (Frage 1 bis 9 waren: Husten, Atemnot, Schwindel, Schlafstörung, Schwäche, Appetitmangel, Herzstolpern, Unwohlsein) sollten wie auch die folgenden Fragen in ihrer Ausprägung gewichtet werden (Fragen 10 bis 25).

Der Fragebogen wurde von PRG entwickelt, die englische Übersetzung durch AJC angepasst. Der SAQOL-Fragebogen wurde konstruiert für lungenerkrankte Patienten mit und ohne Malignom.

\section{SAQOL-Fragebogen, Konstruktion und Domänen}

Der Fragebogen „Selbsteinschätzung der Lebensqualität (SAQOL)“ besteht aus 27 Fragen in 5 Domänen, sie beinhalten Symptome (Husten, Atemnot, Schwindel, Schlafstörung, Schwäche, Appetitmangel, Herzstolpern, Unwohlsein), soziale und physische Mobilität (Aktivitäten des täglichen Lebens, Möglichkeit Hobbys nachzugehen, das Haus zu verlassen, verminderte Aktivitäten, das Empfinden über die krankheitsbedingten Limitationen im Alltag, Kontakte zu Freunden und Verwandten, Anzahl bewältigter Treppenstufen), psychosoziale Auswirkungen (optimistisch, niedergeschlagen und hoffnungslos, Einfluss auf die Stimmung, spirituelle Missempfindung, Alteration des Sexuallebens), Interaktion mit Helfern (die Qualität der medizinischen Versorgung, die Hilfe durch Ärzte und Schwestern, andere nicht gesundheitsassoziierte Umstände) und eine allgemeine Einschätzung (physisches Befinden, generelle Lebensqualität).

\section{Auswerteregeln des SAQOL}

Die Fragen betonen die von den Patienten gewichteten Auswirkungen ihrer Erkrankung auf die Emotion und Wahrnehmung. Dafür wurde eine 4 Punkt Likert-Skala verwendet, die den Vorteil hat, eine Gewichtung ober- und unterhalb der Mitte zu fordern und damit die bekannte „zentrale Tendenz“ der Fragebogenbeantwortung zu vermeiden. Die beiden letzten Fragen sollten eine feinere Graduierung erfahren. Sie wurden mit einer 7 Punkt Likert-Skala ausgestattet (physisches Befinden, generelle Lebensqualität), der einen Wertebereich von kompletter Abwesenheit einer Beschwerde (0 Punkte) bis zu einer vollen Belastung (100 Punkte) umfasst, wobei ein höherer Wert eine höhere Beeinträchtigung der Lebensqualität widerspiegelt. Die Abstände zwischen Punkten der Likert-Skala repräsentieren gleiche Ab- 
stände der Veränderung. Zur Sicherstellung der internen Validität hatte der Fragebogen eine zweiseitige Gewichtung. Somit repräsentierte für einen Teil der Fragen der erste Punkt eine volle Belastung (100\%, Fragen 1-9, $11-13,15,18,20-22)$ und für den Rest der letzte Punkt.

\section{Validität, Reliabilität, Test- und Retest-Stabilität}

Nach den Vorgaben sollte in erster Linie auf eine organbezogene (Lungen-) Tumormanifestation und in zweiter Linie auf eine Lungenerkrankung, besonders auf COPD geprüft werden, da ein großer Teil unserer Patienten mit beiden Erkrankungen belastet ist. Als Tumorfragebogen zogen wir den QLQ C-30 der EORTC, in der deutschen Version, der in einer Teilgruppe von Patienten mit weit fortgeschrittenem BC (UICC [15]) bereits eingesetzt wurde $[13,14]$. Wir nahmen an, dass in dieser Subgruppe, die beide Fragebogen zum selben Zeitpunkt in randomisierter Reihenfolge ausgefüllt hatten, mit entsprechender Auswertungsanpassung (schlechteste Lebensqualität hat 100 Punkte) ein Vergleich als Bestandteil der Validität herangezogen werden kann. Begrenzungen ergaben sich aus dem Charakter des QLQ C-30, der als Basisfragebogen gilt und mit einem (im Zeitraum der Studie nicht verfügbaren) Zusatzmodul für die Lunge kombiniert werden muss mit weiteren 12 Fragen [8].

\section{Lebensqualität bei Patienten mit schwerer COPD und Sauerstofflangzeittherapie (LOT)}

Um die Validität bei Patienten mit schwerer COPD zu prüfen, konnten wir die englische Fassung des SAQUOL (unabhängige Vorwärts und Rückwärtsübersetzung, Machbarkeitsüberprüfung und Anpassung) bei muttersprachlich englisch sprechenden Patienten anwenden. In dieser zusätzlichen Gruppe $(n=20)$ wurde gemeinsam mit dem SAQOL (englische Version) ein in Englisch konzipierter lungenspezifischer Fragebogen am Flinders Medical Centre, Adelaide, South Australia (AJC) ausgegeben. Diese Gruppe ist Bestandteil einer prospektiven longitudinalen Studie, die den Einfluss einer Sauerstofflangzeittherapie auf die Lebensqualität von Patienten mit schwerer COPD evaluiert. Die Studie erfasst Patienten mit der primären Diagnose einer schweren COPD, jünger als 80 Jahre, für die die Indikation zur Sauerstofflangzeittherapie besteht und die in der Lage sind, den Fragebogen auszufüllen. Patienten mit schwerer psychischer Störung oder mit gleichzeitiger Tumormanifestation wurden ausgeschlossen. Diesen Patienten wurden neben physiologischen Messungen vier validierte Lebensqualitätsfragebogen vorgelegt, das Nottingham Health Profile (NHP) [16], der Medical Outcomes Short Form 36 item Questionnaire (MOS SF-36) [17] und der krankheitsspezifische Chronic Respiratory Disease Questionnaire (CRQ) $[18,19]$. Hiervon wurde eine Subgruppe von 20 Patienten gebeten, in zufälliger Reihenfolge den SAQOL (englische Version) zu beantworten.

\section{Test-/Retest-Stabilität}

Die Retest-Stabilität wurde in einer Untergruppe der Patienten $(n=42)$ mit stabiler Erkrankung (in den letzten drei Monaten keine Medikamentenänderung, keine Exazerbationen) geprüft, die den Fragebogen während einer zweiten Visite ausfüllten (28 Tage $\pm 2,3$ Tage SEM).

\section{Patienten}

Der neue Lebensqualitätsfragebogen „Selbsteinschätzung der Lebensqualität (SAQOL)“ wurde in einer prospektiven offenen Pilotstudie in der Thoraxchirurgischen Ambulanz und der pneumologischen Ambulanz der Albert-Ludwigs-Universität Freiburg ausgegeben (PRG). Es wurden 93 ambulante Patienten mit Lungenkrebs befragt (18 Frauen, 75 Männer, im Mittel 62,6 Jahre, von 18 bis 81 Jahre), 54 davon waren im Stadium IIIB oder IV eines Bronchialkarzinoms, außerhalb eines operativen/kurativen Therapieansatzes (Tumorstadien gemäß UICC [15]). Diese Gruppe mit fortgeschrittenem Tumorstadium nahm an einer prospektiven Multizenter-Studie der EORTC teil [13]). In dieser Studie lag das Interesse auf der Korrelation vom individuellen Wert der Lebensqualität (QLQ C-30) auf die Überlebenszeit bei fortgeschrittenen Tumoren (jeder Histologie) $(n=835)$. [13] Von dieser teilnehmenden Gruppe wurden nach dem Zufallsprinzip erst der QLQ C-30 ( $n=27)$ oder der SAQOL ( $n=27)$ als erster Fragebogen ausgehändigt. Von allen Patienten hatten 54 schwere COPD (Messung und Klassifikation nach ERS und ATS) [17,20]. Alle Patienten waren älter als 18 Jahre und konnten selbständig den Fragebogen lesen und ausfüllen. Eine histologische Sicherung einer pulmonalen Raumforderung, planare Röntgenuntersuchung des Thorax, Computertomographie des Thorax und Lungenfunktion waren für jeden Teilnehmer verfügbar.

Die kumulativen und vergleichbaren Werte der einzelnen Fragen und vergleichbarer Domänen beider Fragebogen wurden der Auswertung zugrunde gelegt. Die klinischen Parameter wurden mit den Werten mit den Werten im SAQOL korreliert. Besonderes Augenmerk wurde auf die Auswirkungen einer begleitenden COPD gelegt.

Die Studie wurde nach Aufklärung und mit vollem Einverständnis der Patienten durchgeführt, unter Beachtung der ethischen Standards, gemäß des Votums der örtlichen Ethikkommission. Die Patientendaten und Befunde wurden nach den üblichen Standards der Europäischen Respiratorischen Gesellschaft und der American Thoracic Society gewertet [17,20]. Die Tumorstadien wurden gemäß der UICC qualifiziert. [15]

\section{QLQ-C30 Basisfragebogen der EORTC}

Der QLQ-C30 ist ein etabliertes 30-Frageninstrument für Tumorpatienten, bestehend aus Mehrfach- und Einfachfragen zur multifunktionalen Messung der Lebensqualität. Die fünf funktionalen Skalen umfassen physischen Zustand, Rollenverhalten, kognitiv, emotional und sozial. Die drei abgefragten Symptomskalen betreffen Ermüdung, Schmerz, Schwindel und Erbrechen. Zwei weitere Skalen zielen auf globale Gesundheit und Lebensqualität. Die später verfügbaren Ergänzungen als Lungenmodul waren zum Zeitpunkt der Studie noch nicht verfügbar (12 weitere Fragen). Eine Teilgruppe von 54 Patienten war Teil einer Multizenterstudie zur Erhebung der Lebensqualität [13], in der der Einfluss der Lebensqualität auf die Überlebenszeit untersucht wurde. In dieser Untergruppe wurden die beiden Fragebogen SAQOL und QLQ-C30 verglichen. 


\section{Statistik}

Mit vergleichbarem Bewertungssystem wurden die Frageninterkorrelationen, die Korrelationen zu den klinischen Parametern (Histologie, TNM-Stadium, Alter, Geschlecht, weitere Diagnosen) und Befunde (Ganzkörperplethysmographie) über den t-Test für paarige Proben und zwischen den Fragen durch den Test auf Linearität und die Spearman Rangordnungskorrelation berechnet. Die Überlebenszeit wurde evaluiert über die Kaplan-Meier Prozedur. Interne Konsistenz wurde über den Cronbach's Alpha Koeffizienten ermittelt. Retest-Stabilität wurde über den Pearson's Korrelationskoeffizienten geprüft. Die Daten wurden mit dem Programm SPSS-WIN PC ${ }^{\circledR} 12.0$ analysiert.

\section{Ergebnisse}

\section{Klinische Daten}

Der SAQOL wurde von 93 Patienten (18 Frauen, 75 Männer, im Mittel 62,6 Jahre, von 18 bis 81) mit pulmonalen Malignomen ausgefüllt. Bronchialkarzinome wurden in 75\% diagnostiziert als undifferenziertes nichtkleinzelliges Karzinom (35,5\%), Plattenepithelkarzinom (11,8\%), großzelliges Karzinom (8,6\%), Adenokarzinom (10,8\%) und kleinzelliges Karzinom (7,5\%). Die pulmonale Metastasierung bei fünfundzwanzig Prozent der Patienten hatte ihren Ursprung im Kolon (25\%) und der Niere (75\%). Von Lungenkrebs ausgehende Metastasierungsorte waren die Knochen (5,4\%), das Gehirn (4,3\%), die Leber (3,2\%), die Pleura $(3,2 \%)$ und in mehreren Metastasierungsorten (6,5\%). Tumorinfiltration wurde gefunden in die peribronchiale Umgebung (5,4\%), in die ipsilateral-paratrachealen $(14,0 \%)$ und kontralateralen (9,7\%) Lymphknoten. Eine Zuordnung in ein T-Stadium konnte für zwei Drittel der Patienten vorgenommen werden: $\mathrm{T}_{0}$ $(2,2 \%), \mathrm{T}_{1}(6,5 \%), \mathrm{T}_{2}(20,4 \%), \mathrm{T}_{3}(8,6 \%)$ and $\mathrm{T}_{4}(18,3 \%)$.

\section{SAQOL-Fragebogen, Konstruktion und Domänen}

Die Reliabilitätsanalyse zeigte in den Domänen ein Cronbach's Alpha von 0,91 (standardisiert 0,92). Mit diesem Modell entspricht dies einer sehr guten inneren Konsistenz, ermittelt über die durchschnittliche Inter-Item-Korrelation.

In den einzelnen Domänen zeigten sich überwiegend Mittelwerte und Standardabweichungen, die auf eine nur leicht- bis mittelgradige Einschränkung der Lebensqualität schließen lassen: Der Bereich soziale und körperliche Mobilität wies 41,6 Punkte auf (SD: 17,7), für psychosoziale Beeinträchtigungen wurden 37,7 Punkte ermittelt (SD: 25,3), für symptombezogene Beeinträchtigung 36,5 Punkte (SD: 25,7) und die generelle physische und psychische Lebensqualität 37,5 Punkte (SD: 17,7).

Die Retest-Stabilität wurde über den Partialkorrelationskoeffizienten ermittelt (Pearsons) und konnte für alle gemessenen Bereiche mit sehr guten Ergebnissen errechnet werden: Helferinteraktion $0,66(\mathrm{p}=0,001)$, soziale und physische Mobilität 0,56 $(p=0,006)$, psychosoziale Beeinträchtigung $0,46 \quad(p=0,02)$, symptombezogene Beeinträchtigung $0,44(p=0,03)$, generelle Lebensqualität $0,66(\mathrm{p}=0,001)$ und im Mittel aller Fragen 0,74 $(\mathrm{p}=0,0001)$.
Tab. 1 SAQOL: Korrelation zu QLQ-C30 in 5 Domänen in den Stadien IIIB und IV des Bronchialkarzinoms

\begin{tabular}{|c|c|c|c|c|c|}
\hline QLQ-C30 & $\begin{array}{l}\text { SAQOL } \\
\text { Symptome }\end{array}$ & $\begin{array}{l}\text { soziale und } \\
\text { physische } \\
\text { Mobilität }\end{array}$ & $\begin{array}{l}\text { psycho- } \\
\text { soziale Beein- } \\
\text { trächtigung }\end{array}$ & $\begin{array}{l}\text { Helfer- } \\
\text { inter- } \\
\text { aktion }\end{array}$ & $\begin{array}{l}\text { allgemeine } \\
\text { Selbstein- } \\
\text { schätzung }\end{array}$ \\
\hline physisch & $0,7^{* * *}$ & $0,5^{* * *}$ & $0,6^{* * *}$ & $0,4^{* *}$ & $0,5^{* * *}$ \\
\hline Rolle & $0,7^{* * *}$ & $0,4^{* *}$ & $0,3^{* *}$ & $0,2^{* * *}$ & $0,3^{* * *}$ \\
\hline emotional & $0,3^{* *}$ & $0,2^{* * *}$ & $0,54^{* * *}$ & $0,3^{* * *}$ & $0,4^{* *}$ \\
\hline kognitiv & $0,4^{* *}$ & $0,3^{* * *}$ & $0,4^{* * *}$ & $0,2^{* * *}$ & $0,3^{* * *}$ \\
\hline $\begin{array}{l}\text { soziales } \\
\text { Funktionieren }\end{array}$ & $0,3^{* *}$ & $0,5^{* * *}$ & $0,3^{* *}$ & $0,4^{* *}$ & $0,7^{* * *}$ \\
\hline allgemein & $0,6 * * *$ & $0,4^{* *}$ & $0,5^{* * *}$ & $0,4^{* *}$ & $0,6^{* * *}$ \\
\hline
\end{tabular}

In den Spalten: Domänen des QLQ-C 30 der EORTC, in den Zeilen: Domänen des SAQOL. Korrelationen zwischen den Domänen über den Spearman Korrelationskoeffizienten, ${ }^{*} p=0,05-0,01,{ }^{* *} p=0,01-0,005,{ }^{* * *} p<0,005$, QLQ-C30 wurde für eine vergleichbare Auswertung der Punktwerte adaptiert

\section{Validität des SAQOL bei pulmonalen Malignomen}

Der Tab. 1 können Interkorrelationen von SAQOL zum QLQ C-30 der EORTC bei Tumorpatienten entnommen werden. Die für den bilateralen Vergleich angepassten Domänen zeigen hierbei gute bis sehr gute Korrelationen. Die höchsten Signifikanzniveaus wurden in Fragen nach Symptomen, allgemeine Selbsteinschätzung, generelle physische und psychische Beeinträchtigung, psychosoziale Auswirkungen gefunden. Schwächere Korrelationen wurden innerhalb der Domänen ermittelt, die der Interaktion mit Ärzten und Schwestern sowie sozialen und physischen Mobilität gewidmet und die für Patienten mit Lungenerkrankungen wichtig sind.

\section{Lebensqualität, Auswirkungen bei fortgeschrittenem Tumorstadium}

Patienten mit potenziell kurablem pulmonalem Tumor hatten eine leicht- bis mittelgradige Lebensqualitätsbeeinträchtigung. Die 54 Patienten mit fortgeschrittenen Tumorstadien, überwiegend ohne eine Option zur kurativen Behandlung wiesen dagegen eine deutlichere Lebensqualitätsbeeinträchtigung auf. Bei sonst guten Korrelationen zum QLQ-C30-Fragebogen reduzierten zum Teil weite Varianzbereiche das Signifikanzniveau (Tab. 2).

Die Ergebnisse beider Fragebogen zeigten gleichermaßen, dass das individuelle Ausmaß der Lebensqualitätsbeeinträchtigung nicht abhängig vom Tumorstadium war. Zum Beispiel hatten Patienten im Tumorstadium I mit einem mittleren Punktwert von 49,9 in der Domäne „soziale Interaktion“ des QLQ C-30 eine bedeutendere Lebensqualitätsverschlechterung angegeben als Patienten mit einem Stadium IV Karzinom mit einem mittleren Punktewert von 41,1. Hier zeigt sich die bekannte Unabhängigkeit der Lebensqualitätmessung von den klinischen Beurteilungsmustern (Abb.1). 
Tab. 2 Lebensqualität: Ausmaß der Einschränkung für Patienten in verschiedenen Tumorstadien des Bronchialkarzinoms

\begin{tabular}{lllllll}
\hline \multicolumn{7}{c}{ SAQOL (mittlerer Score-Wert, SEM) } \\
$\begin{array}{l}\text { T-Sta- } \\
\text { dium }\end{array}$ & Symptome & $\begin{array}{l}\text { soziale/ } \\
\text { physische } \\
\text { Mobilität }\end{array}$ & $\begin{array}{l}\text { zsale Beein- } \\
\text { trächtigung }\end{array}$ & $\begin{array}{l}\text { Helferinter- } \\
\text { aktion }\end{array}$ & $\begin{array}{l}\text { allgemeine } \\
\text { Selbstein- } \\
\text { schätzung }\end{array}$ \\
\hline IV & $45,4(20,5)$ & $38,8(23,5)$ & $46,8(27,9)$ & $41,1(22,9)$ & $48,7(13,1)$ \\
\hline III & $52,8(21,9)$ & $53,8(22,0)$ & $54,8(14,6)$ & $52,3(21,2)$ & $54,3(13,0)$ \\
\hline II & $38,3(22,2)$ & $42,7(17,2)$ & $42,0(18,3)$ & $38,3(29,3)$ & $41,5(16,2)$ \\
\hline I & $48,8(38,3)$ & $50,9(24,8)$ & $54,8(14,6)$ & $40,2(33,0)$ & $52,0(22,0)$ \\
\hline 0 & $49,9(39,2)$ & $41,2(21,8)$ & $46,8(27,9)$ & $37,5(53,0)$ & $42,8(31,8)$ \\
\hline
\end{tabular}

\begin{tabular}{llllll}
\hline \multicolumn{7}{c}{$\begin{array}{l}\text { QLQ-C30 (mittlerer Score-Wert, SEM) } \\
\text { psycho- } \\
\text { T-Sta- } \\
\text { dium }\end{array}$} & $\begin{array}{l}\text { Symptome } \\
\text { sozial }\end{array}$ & $\begin{array}{l}\text { Mobilität } \\
\text { soziale } \\
\text { Interaktion }\end{array}$ & $\begin{array}{l}\text { allgemeine } \\
\text { Selbstein- } \\
\text { schätzung }\end{array}$ \\
\hline IV & $48,3(27,5)$ & $53,5(15,3)$ & $37,6(17,5)$ & $41,1(40,0)$ & $46,8(15,9)$ \\
\hline III & $41,7(20,7)$ & $49,7(22,1)$ & $40,9(26,7)$ & $37,4(27,8)$ & $44,6(20,7)$ \\
\hline II & $30,2(21,2)$ & $33,9(18,5)$ & $32,9(19,5)$ & $31,5(35,9)$ & $33,2(15,6)$ \\
\hline I & $35,2(23,5)$ & $29,7(31,5)$ & $35,5(27,5)$ & $49,9(45,9)$ & $34,1(25,8)$ \\
\hline 0 & $61,0(-,-)$ & $15,1(17,1)$ & $6,6(6,6)$ & $16,6(23,5)$ & $29,2(-,-)$ \\
\hline
\end{tabular}

In den Spalten Symptom-QOL-Werte von unterschiedlichen Tumorstadien des Bronchialkarzinoms, in den Zeilen: bezogen auf die Domänen, zuerst der QLQ-C30 der EORTC und dann der SAQOL. In Klammern Standardabweichung.

Im Tumorstadium IIIB and IV wurden mit dem SAQOL ähnliche Punktwerte ermittelt wie mit dem QLQ C30, der gerade hierfür ausführlich evaluiert wurde.

\section{Lebensqualität als prognostischer Faktor}

Der Median aller Patienten über alle Fragen beträgt 42,1 Punkte. Wurde dieser Wert zur Unterteilung herangezogen, zeigten die beiden Gruppen mit einer Lebensqualität unterhalb dieses Wertes eine signifikant kürzere mediane Überlebenszeit von 17 Monaten (CI 14 bis 20 Monate) als die Gruppe oberhalb dieses Wertes mit einer medianen Überlebenszeit von 55 Monaten (CI 22 bis 88 Monate ) über den Log Rank Test ermittelt $(p=0,05)$, ohne bedeutsame Unterschiede im Alter oder den Tumorstadien. Dies gilt für beide untersuchten Fragebogen und wurde in derselben Gruppe mit dem QLQ C30 im Rahmen einer Multizenterstudie evaluiert [13]. Für den SAQOL lässt sich die Kaplan-MeierKurve in Abb. 2 entnehmen.

\section{Die Rolle der chronischen Atemwegsobstruktion für die Lebensqualität}

Zunehmende $\mathrm{FEV}_{1}$-Limitierung ließ sich als lebensqualitätsmindernd im SAQOL und auch im QLQ-C30 nachweisen, dort jedoch mit geringerer Ausprägung. Wie der Tab. 3 zu entnehmen ist, zeigt der $\mathrm{FEV}_{1}$-Verlust wegen einer COPD sich in einem negativen Spearman Korrelations-Koeffizienten in den Domänen „Symptome“, „Soziale Beeinträchtigung“, „Psychologische Auswirkung“, „Physische und soziale Mobilität“, „Allgemeine physische Selbsteinschätzung“ und „allgemeine Lebensqualität“. Bemerkenswert ist im QLQ C-30 der Mangel an signifikanten Korrelationen. Der Tab. 4 kann zudem für den QLQ C-30 keine Korrela-

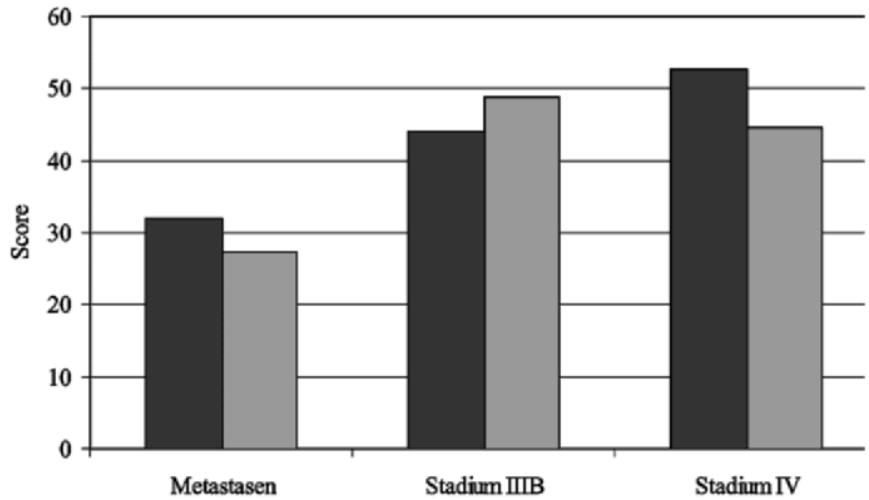

Abb. 1 Lebensqualität: Beeinträchtigung für Patienten mit Bronchialkarzinom im Stadium IIIB und IV, Korrelation SAQOL (schwarze Säulen) zu QLQ-C30 (graue Säulen): kein signifikanter Unterschied.

Die durchschnittlichen Gesamtscores beider Fragebögen, QLQ-C30 und SAQOL, werden verglichen: Es fand sich kein signifikanter Unterschied in den Korrelationen zu den Stadien IIIB und IV des Bronchialkarzinoms, bzw. bei Metastasierung in die Lunge.

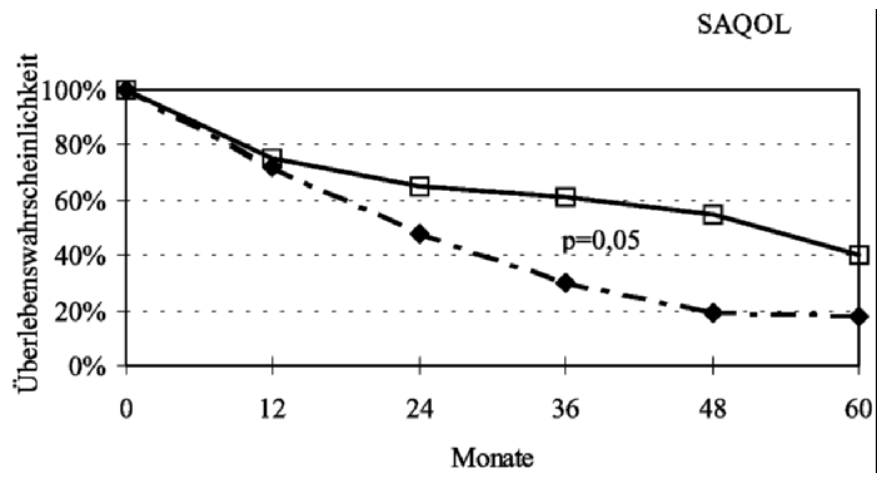

Abb. 2 Lebensqualität (bessere: durchgezogene Linie, geringere: unterbrochene Linie) als prognostischer Faktor für Patienten mit Bronchialkarzinomen der Stadien IIIB und IV.

In einer Untergruppe mit inoperablem Bronchialkarzinom wurde die eine Hälfte mit einem Labensqualitätswert besser („besser“) als der Median aller Werte $(n=27)$ mit der anderen Hälfte, die unterhalb des Medianes lagen verglichen („schlechter“): Eine signifikant bessere Überlebenszeit war bei Gleichverteilung sonstiger Parameter mit besserer Lebensqualität verbunden.

tion zu den Lebensqualitätswerten bei Hypoxämie und leichter Hyperkapnie entnommen werden.

Die Anwesenheit einer chronischen Atemwegsobstruktion hatte größere Auswirkungen auf die Lebensqualität als zum Beispiel unterschiedliche Tumorstadien. Selbst im zwar allgemein auf Tumor, jedoch nicht auf das Organ Lunge spezifizierten QLQ C-30 deutet sich der Einfluss einer Atemwegsobstruktion auf eine Lebensqualitätsverschlechterung nur an. Im spezifischeren SAQOL drückt sich dieser Einfluss besser aus (Tab. 4).

Eine zusätzliche Gruppe von 20 Patienten (Männer/Frauen: 11/9) füllte den SAQOL Fragebogen aus. Diese Patienten benutzten entweder bereits Langzeitsauerstofftherapie (LOT), $\mathrm{n}=16$ oder waren dafür vorgesehen, $n=4$. Die Patienten waren $68,3 \pm 6,7$ Jahre 
Tab. 3 Korrelation einer FEV 1 -Limitierung zu SAQOL und QLQ-C30 (Spearman Korrelationkoeffizient)

\begin{tabular}{|c|c|c|c|c|}
\hline Item & SAQOL & & QLQ-C30 & \\
\hline Symptome & $-0,26$ & $p=0,01$ & $-0,16$ & $p=0,5$ \\
\hline soziale Beeinträchtigung & $-0,27$ & $p=0,008$ & $-0,20$ & $p=0,001$ \\
\hline psychologische Auswirkung & $-0,21$ & $p=0,001$ & $-0,003$ & $p=0,9$ \\
\hline $\begin{array}{l}\text { physische und soziale } \\
\text { Mobilität }\end{array}$ & $-0,25$ & $p=0,001$ & 0,16 & $\mathrm{p}=0,1$ \\
\hline $\begin{array}{l}\text { allg. physische Selbstein- } \\
\text { schätzung und allgemeine } \\
\text { Lebensqualität }\end{array}$ & $-0,25$ & $p=0,001$ & $-0,018$ & $p=0,8$ \\
\hline
\end{tabular}

Für die beiden Fragebogen wurde mittels Spearman-Korrelationskoeffizienten die Korrelation einer $\mathrm{FEV}_{1}$-Limitierung zu den Lebensqualitätswerten in den Domänen, jeweils daneben steht das Signifikanzniveau.

Tab. 4 QOL -Werte für chronisch-obstruktive Lungenerkrankung, Korrelation zur Lungenfunktion (Spearman Korrelationskoeffizient)

$\begin{array}{llll}\text { QLQ-C30 } & & & \\ \text { Symptome } & \begin{array}{l}\text { soziale und } \\ \text { physische } \\ \text { Mobilität }\end{array} & \begin{array}{l}\text { psycho- } \\ \text { soziale Be- } \\ \text { einträchti- } \\ \text { gung }\end{array} & \begin{array}{l}\text { Helferinter- } \\ \text { allgemeine } \\ \text { Selbstein- } \\ \text { schätzung }\end{array} \\ & & & \end{array}$

ohne COPD $\quad 35,2(27,0) \quad 40,8(16,8) \quad 29,3(16,8) \quad 24,0(33,4) \quad 35,8(16,4)$

mit COPD $\quad 46,3(24,6) \quad 48,7(18,0) \quad 37,0(17,3) \quad 46,2(38,1) \quad 44,3(16,2)$ SAQOL

ohne COPD $\quad 30,7(20,9) \quad 37,3(20,2) \quad 38,4(17,8) \quad 45,5(31,6) \quad 36,5(14,9)$ mit COPD $\quad 52,2(26,2) \quad 60,4(16,1) \quad 52,4(17,8) \quad 49,2(23,9) \quad 53,1(16,3)$ Spearman Korrelationskoeffizient

\begin{tabular}{|c|c|c|c|c|c|}
\hline$F E V_{1}$ & $-0,26$ & $-0,43$ & $-0,21$ & $-0,27$ & $-0,34$ \\
\hline Sign. & 0,01 & 0,0001 & 0,04 & 0,008 & 0,001 \\
\hline
\end{tabular}

Lebensqualitätswerte in unterschiedlichen Domänen (Zeilen) in Relation zum Vorhandensein oder Abwesenheit einer (COPD): höhere QOL-Beeinträchtigung mit COPD, deutlichere Werte mit SAQOL, zusätzlich nur bei SAQOL erkennbare Korrelation zur Lungenfunktion.

alt. Im SAQOL korrelierte der soziale Mobilität Punktwert signifikant negativ ( $p=0.01$ ) mit dem SF-36 „physisches Funktionieren“, „soziales Funktionieren“ und „generelle Gesundheitswahrnehmung“ und mit der „Vitalität“, der Symptom-Punktewert war signifikant negativ korreliert mit der SF36 Domäne für „physisches Funktionieren“. Eine signifikant positive Korrelation fand sich zwischen dem Punktwert der SAQOL-Domäne „soziale Mobilität“ und „psychosozial“, „emotionale Reaktion“ und „physische Mobilität“. Zudem konnten positive Korrelationen zwischen dem SF-36 und dem NHP in der Domäne „soziale Isolation“ und zwischen der SAQOL-Domäne „allgemeine Lebensqualität“ und NHP „emotionale Reaktionen“ ermittelt werden. Der SAQOL Symptom-Punktewert war signifikant korreliert mit der CRQDomäne „Ermüdung“ und den „Bewältigungsdimensionen“, im weiteren „soziale Mobilität“ mit CRQ „Dyspnoe“ und „Bewältigung“, weiterhin die SAQOL-Domäne „allgemeine Lebensqualität“ mit CRQ „emotionales Funktionieren“ und „Bewältigung“. Der Vergleich mit dem für COPD-Patienten besonders verlässli-
Tab. 5 Korrelation zwischen Nottingham Health Profile und SAQOL bei COPD-Patienten mit LOT $(n=20)$ (Spearman Korrelationskoeffizient)

\begin{tabular}{|c|c|c|c|c|c|}
\hline $\begin{array}{l}\text { Nottingham } \\
\text { Health } \\
\text { Profile }\end{array}$ & $\begin{array}{l}\text { SAQOL } \\
\text { Symp- } \\
\text { tome }\end{array}$ & $\begin{array}{l}\text { soziale und } \\
\text { physische } \\
\text { Mobilität }\end{array}$ & $\begin{array}{l}\text { psychosoziale } \\
\text { Beeinträchti- } \\
\text { gung }\end{array}$ & $\begin{array}{l}\text { Helferinter- } \\
\text { aktion }\end{array}$ & $\begin{array}{l}\text { allgemeine } \\
\text { Selbstein- } \\
\text { schätzung }\end{array}$ \\
\hline Energie & 0,24 & 0,44 & 0,30 & 0,12 & 0,12 \\
\hline Schmerz & 0,17 & 0,26 & 0,18 & 0,09 & $-0,04$ \\
\hline $\begin{array}{l}\text { emotionale } \\
\text { Reaktionen }\end{array}$ & 0,45 & $0,63^{*}$ & 0,49 & 0,10 & $0,61^{*}$ \\
\hline Schlaf & 0,37 & 0,10 & 0,13 & $-0,12$ & 0,36 \\
\hline $\begin{array}{l}\text { soziale } \\
\text { Isolation }\end{array}$ & 0,23 & 0,56 & $0,76^{* *}$ & 0,10 & 0,48 \\
\hline $\begin{array}{l}\text { physische } \\
\text { Mobilität }\end{array}$ & 0,31 & $0,59 *$ & 0,50 & $0,56^{*}$ & 0,15 \\
\hline
\end{tabular}

Lebensqualitätswerte in der Untergruppe mit schwerer COPD und der Erfordernis einer Sauerstofflangzeittherapie (LOT) ohne Malignom: Gut vergleichbare Domänen zwischen Nottingham Health Profile und SAQOL. *: $p<0,01$, ${ }^{* *}$ : $\mathrm{p}<0,001$ (Spearman Rangordnungs-Korrelation).

chen Nottingham-Health-Profile, mit dem hier vorgestellten SAQOL zeigt in den entsprechenden Domänen gute Korrelationen (Tab. 5).

\section{Diskussion}

Trotz einer scheinbaren Vielzahl an Instrumenten zur Lebensqualitätsevaluierung gibt es den Bedarf für einen Fragebogen, der valide ist für Patienten mit pulmonaler Erkrankung mit oder ohne Malignom. Patienten mit pulmonalen Tumoren leiden zusätzlich oft an chronisch obstruktiver Atemwegserkrankung, so dass ein Instrument zur Messung der Lebensqualität die obstruktionsbedingten Beeinträchtigung einbeziehen sollte $[7,9,12]$. Für das Messinstrument muss darüber hinaus gelten, durch höchstmögliche Kürze und geringen Zeitaufwand eine hohe Patientenakzeptanz zu erreichen. Zum Beispiel sollten nicht mehr als dreißig Fragen zu beantworten sein. Dies schließt unspezifische und durch vielfältige Fragen umfangreiche Fragebogen [8] oder aber die Ausgabe von zwei separaten Fragebogen für Krebserkrankung und COPD aus. Der hier vorgestellte SAQOL, ein für Patienten mit Atemwegserkrankung oder/und Malignom konzipierter Fragebogen, wurde von unseren ambulanten Patienten gut angenommen. Der selbst auszufüllende Fragebogen erforderte nicht mehr als 10 Minuten zur Bearbeitung. Der Fragebogen soll im Alltag eingesetzt werden können. Er wurde im typischen Ambulanzsetting konzipiert, mit der Rückmeldung von Kollegen und Patienten bis zu seiner hier vorgestellten Form modifiziert, die sich auf die Hauptthemen des Patienteninteresses konzentriert. [21-23].

Im Vergleich zu einem in Europa vielfach eingesetzten Tumorfragebogen, dem QLQ C30 der EORTC konnte sich der vorgestellte Fragebogen als valide bestätigen, gerade in der Gruppe der Patienten mit fortgeschrittenem, inkurablem Bronchialkarzinom, bzw. nicht operablen pulmonalen Metastasen. Diese Gruppe nahm zur Evaluierung des QLQ-C30 an einer europäischen Mul- 


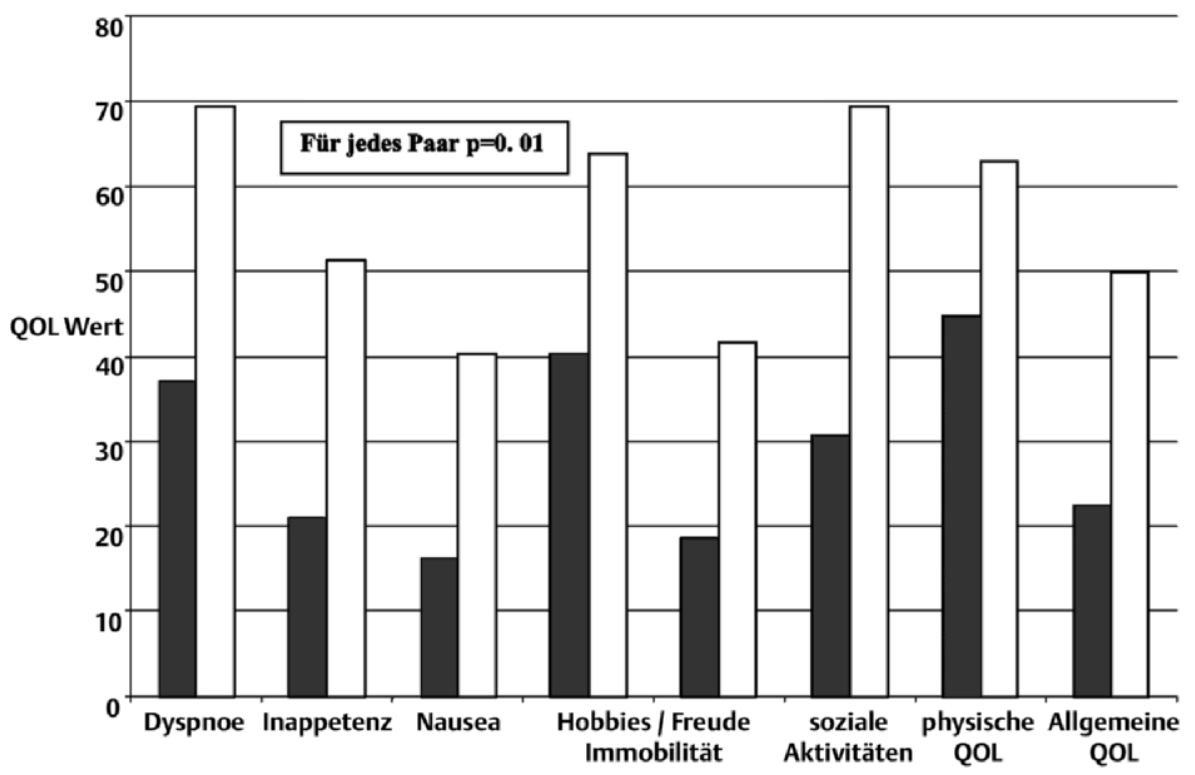

Abb. 3: Lebensqualität (SAQOL) bei Patienten mit (weiße Säule) und ohne (schwarze Säule) COPD in fortgeschrittenen Stadien des Bronchialkarzinoms.

Der Nachweis einer COPD hat bei Patienten mit Bronchialkarzinom signifikanten Einfluss auf die durch SAQOL gemessenen Lebensqualitätswerte in jeder Domäne. tizenterstudie der EORTC teil (inklusive Australien und Canada) [13]. Der hier neu vorgestellte SAQOL konnte bei kürzerem Umfang sich hier als ebenbürtig bewähren. Aber auch bei den Patienten in geringeren Tumorstadien konnte in den vergleichbaren Domänen der beiden evaluierten Fragebogen gute Korrelationen gemessen werden.

Im Ergebnis zeigt der kürzere SAQOL Reliabilität, Retest-Stabilität und vor allem Validität. Es stellt sich die Frage, inwieweit der neue SAQOL gegenüber etablierten Fragebogen eine Erweiterung der Ergebnisse aufweist und ob er spezifische Unterschiede zu den vorhandenen Fragebogen darstellen kann. Hierzu identifizierten wir zum Beispiel einen moderierenden Faktor der Lebensqualität bei unseren Patienten, der sich im Vergleich zum etablierten QLQ-C30 mit dem SAQOL deutlicher abbilden lässt: Lungenfunktionsbeeinträchtigung aufgrund chronisch-obstruktiver Atemwegsobstruktion. Dieser messbare Parameter hat Einfluss auf eine höhere Beeinträchtigung bei Patienten mit COPD im Vergleich zu Patienten ohne chronisch-obstruktiver Atemwegserkrankung (s. Abb.3).

Auch bei Patienten ohne BC, bei denen eine schwere COPD zu respiratorischer Insuffizienz geführt hat mit der Erfordernis einer Sauerstofflangzeittherapie, konnte der Fragebogen gegenüber dem ausführlich evaluierten und weltweit eingesetzten, besonders für COPD relevanten und gerade die psycho-sozialen Alterationen am besten abbildenden Nottingham Health Profile [13] gute Korrelationen zeigen.

\section{Einfluss der chronisch obstruktiven Lungenerkrankung auf die Lebensqualität}

Patienten mit pulmonalen Malignomen die auch an einer COPD leiden, geben eine höhere Lebensqualitätsbeeinträchtigung an als Patienten ohne Atemwegserkrankung, auch in vergleichbar fortgeschrittenen Tumorstadien. Signifikant größere Beeinträchtigungen fanden sich bei schwerer Lungenfunktions-Einschränkung $\left(\mathrm{FEV}_{1}, \mathrm{MEF} 50\right)$. Bei diesen Patienten konnte der neue Lebensqualitätsfragebogen eine deutliche Korrelation zum
$\mathrm{FEV}_{1}$-Verlust bei COPD darstellen im Gegensatz zu dem tumor-, jedoch nicht organspezifischen QLQ C-30 der EORTC, deren Lungenmodul mit dreizehn weiteren Fragen zu diesem Zeitpunkt nicht in seiner gültigen Version zur Verfügung stand. Dieser Mangel an Korrelation zur Lungenfunktionsverschlechterung im QLQ C-30 wurde bereits in anderen Studien bemerkt, $[13,18]$ und betont die Forderung nach Organspezifität eines Fragebogens.

Wie bereits in der gemeinsamen Studie mit der EORTC gezeigt, besteht auch in der vorgestellten Studie eine Beziehung zwischen einer Lebensqualitätsbeeinträchtigung und einer signifikant reduzierten Überlebenszeit bei Patienten mit fortgeschrittenem und inkurablem Tumorstadium.[13,21-23] Hier muss der Wert der Lebensqualitätmessung als bedeutender Faktor hervorgehoben werden, besonders bei Patienten die an einer chronischen Atemwegserkrankung leiden. Diese Patienten tragen eine Last, die ihre Prognose limitieren kann, durch den zusätzlichen Einfluss, den eine Lebensqualitätsminderung ausüben kann.

\section{Danksagung}

Besonderer Dank gilt JM Cranston, Respiratory Unit, Department of Medicine, Flinders Medical Centre, die bei der Ausgabe und Evaluation der englischen Version des SAQOL half. 
Im folgenden werden Fragen nach Ihrem gesundheitlichen Befinden der letzten 4 Wochen gestellt. Bitte kreuzen Sie ohne langes Nachdenken das für Sie am ehesten zutreffende Kästchen an. Es gibt dabei keine "richtigen" oder "falschen" Antworten. Selbstverständlich unterliegen Ihre Angaben der ärztlichen Schweigepflicht.

Tragen Sie bitte Ihren Namen und Vornamen ein:

Ihr Geburtsdatum: Das heutige Datum:

Hatten Sie folgende Beschwerden in den letzten 4 Wochen:

\begin{tabular}{|c|c|c|c|c|}
\hline 1. Husten & $\square$ täglich & $\square$ häufig & $\square$ selten & $\square$ nie \\
\hline 2. Atemnot & $\square$ täglich & $\square$ häufig & $\square$ selten & $\square$ nie \\
\hline 3. Übelkeit & $\square$ täglich & $\square$ häufig & $\square$ selten & $\square$ nie \\
\hline 4. Schlafstörungen & $\square$ täglich & $\square$ häufig & $\square$ selten & $\square$ nie \\
\hline 5. Schwäche & $\square$ täglich & $\square$ häufig & $\square$ selten & $\square$ nie \\
\hline 6. Appetitmangel & $\square$ täglich & $\square$ häufig & $\square$ selten & $\square$ nie \\
\hline 7. Herzklopfen & $\square$ täglich & $\square$ häufig & $\square$ selten & $\square$ nie \\
\hline 8. Schwindel & $\square$ täglich & $\square$ häufig & $\square$ selten & $\square$ nie \\
\hline 9. Schmerzen & $\square$ täglich & $\square$ häufig & $\square$ selten & $\square$ nie \\
\hline
\end{tabular}

Wie haben Sie sich in den letzten 4 Wochen gefühlt. Bitte kreuzen Sie bei jeder Frage die für Sie zutreffende Aussage an:

10. Die Verrichtungen des Alltags (Haushalt / Einkaufen / Arbeit) erledige ich ohne Unterstützung.

$\square$ immer $\square$ meistens $\square$ selten $\square$ nie

11. Meine Erkrankung zwingt mich auf vieles zu verzichten, was mir Freude macht.

$\square$ immer $\square$ meistens $\quad \square$ selten $\square$ nie

12. Seit meiner Erkrankung bin ich an meine Wohnung nahezu gefesselt und kann sie kaum verlassen.

$\square$ immer $\square$ meistens $\square$ selten $\square$ nie

13. Wegen meiner Erkrankung mußte ich in meinem Alltag bereits viele Umstellungen vornehmen (z. B. Einschränkung meiner Aktivitäten).

immer $\square$ meistens $\square$ selten $\square$ nie

Selbsteinschätzung der Lebensqualität (SAQOL)

\section{Literatur}

${ }^{1}$ Hansell AL, Walk JA, Soriano JB. What do chronic obstructive pulmonary disease patients die from? A multiple cause coding analysis. European Respiratory Journal 2003; 22: 809-814

${ }^{2}$ Kishi K, Gurney JW, Schroeder DR et al. The correlation of emphysema or airway obstruction with the risk of lung cancer: a matched casecontrolled study. European Respiratory Journal 2002; 19: 1093-1098

${ }^{3}$ Tockman MS, Anthonisen NR, Wright EC et al. The intermittant postive pressure breathing trial group, The John Hopkins lung project for the early detection of lung cancer. Airway obstruction and the risk of lung cancer. Annals of Internal Medicine 1987; 106: 512 - 518

${ }^{4}$ Skillrud DM, Offord KP, Miller RD. Higher risk of lung cancer in chronic obstructive pulmonary disease. A prospective, matched controlled study. Annals of Internal Medicine 1986; 106: 503-507

${ }^{5}$ Gore JM, Brophy CJ, Greenstone MA. How well do we care for patients with end stage chronic obstructive pulmonary disease (COPD)? A comparison of palliative care and quality of life in COPD and lung cancer. Thorax 2000; 55 (12): 1000-1006
${ }^{6}$ Sarna L, Evangelista L, Tashkin D et al. Impact of respiratory symptoms and pulmonary function on quality of life of long-term survivors of non-small cell lung cancer. Chest 2004; 125 (2): 439-445

${ }^{7}$ Jones PW, Baveystock CM, Littlejohns P. Relationships between general health measured with the sickness impact profile and respiratory symptoms, physiological measures, and mood in patients with chronic airflow limitation. Am Rev Respir Dis 1989; 140 (6): 1538- 1543

${ }^{8}$ Bergner M, Bobbitt RA, Carter WB et al. The Sickness Impact Profile: development and final revision of a health status measure. Med Care 1981; 19 (8): 787-805

${ }^{9}$ Crockett AJ, Cranston JM, Moss JR et al. The MOS SF-36 health survey questionnaire in severe chronic airflow limitation: comparison with the Nottingham Health Profile. Qual Life Res 1996; 5 (3): 330-338

${ }^{10}$ Guyatt GH, Berman LB, Townsend M et al. A measure of quality of life for clinical trials in chronic lung disease. Thorax 1987; 42 (10): $773-778$

${ }^{11}$ Schipper H, Clinch J, McMurray A et al. Measuring the quality of life of cancer patients: the Functional Living Index-Cancer: development and validation. J Clin Oncol 1984; 2 (5): $472-483$ 


\section{Die Erkrankung hat eigentlich keinen Einfluß auf mein tägliches Leben.} immer $\square$ meistens $\square$ selten $\square$ nie

15. Seit meiner Erkrankung treffe ich meine Freunde und Bekannten viel weniger. $\square$ immer $\square$ meistens $\square$ selten $\square$ nie

17. Ich schaue der Zukunft mit Zuversicht entgegen.

$\square$ immer $\square$ meistens $\square$ selten $\square$ nie

18. Häufig fühle ich mich bedrückt und mutlos.

immer $\square$ meistens $\square$ selten $\square$ nie

19. Meine Erkrankung hat keinen wesentlichen Einfluß auf meine Stimmung.

$\square$ immer $\square$ meistens $\square$ selten $\square$ nie

20. Seit ich erkrankt bin, habe ich allen Glauben verloren.

\begin{tabular}{|l|}
\hline immer $\quad \square$ meistens $\quad \square$ selten $\quad \square$ nie \\
21. Seit meiner Erkrankung stimmt mein Sexualleben nicht mehr. \\
\hline
\end{tabular}

21. Seit meiner Erkrankung stimmt mein Sexualleben nicht mehr.

\begin{tabular}{|lll|}
\hline immer $\quad \square$ meistens $\quad \square$ selten & $\square$ nie \\
\hline 22. Die medizinische Betreuung läßt zu Wünschen übrig. & \\
$\square$ immer $\quad \square$ meistens $\quad \square$ selten & $\square$ nie
\end{tabular}

\section{Die Erkrankung hat mich schon viel Geld gekostet, dies belastet mich.}

$\square$ immer $\square$ meistens $\square$ selten $\square$ nie

24. Mehr als meine Erkrankung haben mich die Begleitumstände und Auswirkungen der Behandlung beeinträchtigt.

\begin{tabular}{|l|l|}
\hline \multicolumn{1}{|c|}{ immer $\square$ meistens $\square$ selten $\quad \square$ nie } \\
\hline 25. In normalem Tempo kann ich so viele Stockwerke ( Stw.) hochsteigen: \\
$\square$ bis $1 / 2$ Stw. $\quad \square 1 / 2$ bis 1 Stw. $\quad \square 1-2$ Stw.
\end{tabular}

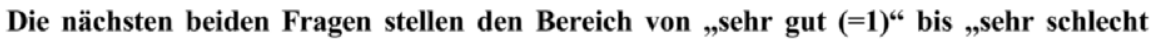

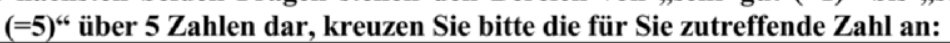

26. Zusammengefasst schätze ich meinen körperlichen Zustand in den letzten 4 Wochen so ein:

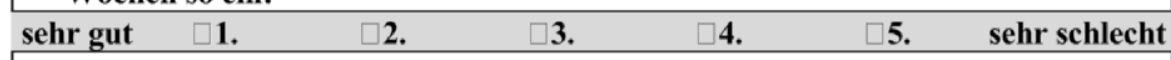

\section{In den letzten 4 Woche habe ich mein Gefühl der Lebensqualität so empfunden:}

sehr gut $\square$. $\quad \square$. $\square$. $\square$. $\square$. $\quad$ sehr schlecht

Vielen Dank für Ihre Mitarbeit!

Geben Sie bitte den ausgefüllten Fragebogen bei Schwestern oder Ärzten ab.

\footnotetext{
Selbsteinschätzung der Lebensqualität (SAQOL)@ Copyright: PR Grahmann 1993
Urheberrechtlich geschützt. Nachdruck und Vervielfalttigungen jeglicher Art, auch einzelner Teile oder Items sowie die Speicherung auf Datenträgern oder die Wiedergabe durch optische oder akustische Medien nur mit schriftlicher Genehmigung.
}

12 Puhan MA, Behnke M, Frey M et al. Self-administration and interviewer-administration of the German Chronic Respiratory Questionnaire: instrument development and assessment of validity and reliability in two randomised studies. Health Qual Life Outcomes 2004; 2 (1): $1-9$

13 Coates A, Porzsolt F, Osoba D. Quality of life in oncology practice: prognostic value of EORTC QLQ-C30 scores in patients with advanced malignancy. Eur J Cancer 1997; 33 (7): 1025-1030

${ }^{14}$ Aaronson NK, Ahmedzai S, Bergman B et al. The European Organization for Research and Treatment of Cancer QLQ-C30: a quality-of-life instrument for use in international clinical trials in oncology. J Natl Cancer Inst 1993; 85 (5): 365 - 376

${ }^{15}$ Hermanek P, Scheibe O, Spiessl B et al. [TNM classification of malignant tumors: a new 1987 edition]. Pathologe 1987; 8 (3): 137-154

${ }^{16}$ Hunt SM, McKenna SP, McEwen J et al. The Nottingham Health Profile: subjective health status and medical consultations. Soc Sci Med [A] 1981; 15 (3 Pt 1): 221 - 229

${ }^{17}$ American Thoracic Society. Standards for the diagnosis and care of patients with chronic obstructive pulmonary disease. American Thoracic Society. Am J Respir Crit Care Med 1995; 152 (5 Pt 2): S77 - 121
18 Stewart AL, Hays RD, Ware JE jr. The MOS short-form general health survey. Reliability and validity in a patient population. Med Care 1988; 26 (7): $724-735$

${ }^{19}$ Wijkstra PJ, TenVergert EM, Altena R van et al. Reliability and validity of the chronic respiratory questionnaire (CRQ). Thorax 1994; 49 (5): $465-467$

${ }^{20}$ Siafakas NM, Vermeire P, Pride NB et al. Optimal assessment and management of chronic obstructive pulmonary disease (COPD). The European Respiratory Society Task Force. Eur Respir J 1995; 8 (8): $1398-1420$

${ }^{21}$ Grahmann PR. Quality of Life in COPD and Bronchogenic Carcinoma. Chest 1996; 110 (4): 42S

${ }^{22}$ Grahmann PR, Kroegel C, Matthys H. Self Assessment Quality of Life Impairment Questionnaire (SAQUOL-Q) and COPD. Schweiz Med Wochenschr 1996; 126 (10 (Supp) 75): $20 \mathrm{~S}$

${ }^{23}$ Grahmann PR, Wertzel H, Berwanger I et al. Quality of life impairment and survival in out-patients with pulmonary tumor and COPD. Monaldi Arch Chest Dis 1995; 50(1): 83A 